\title{
Cardiac nuclear receptors: architects of mitochondrial structure and function
}

\author{
Rick B. Vega and Daniel P. Kelly
}

Cardiovascular Metabolism Program, Center for Metabolic Origins of Disease, Sanford Burnham Prebys Medical Discovery Institute, Orlando, Florida, USA.

\begin{abstract}
The adult heart is uniquely designed and equipped to provide a continuous supply of energy in the form of ATP to support persistent contractile function. This high-capacity energy transduction system is the result of a remarkable surge in mitochondrial biogenesis and maturation during the fetal-to-adult transition in cardiac development. Substantial evidence indicates that nuclear receptor signaling is integral to dynamic changes in the cardiac mitochondrial phenotype in response to developmental cues, in response to diverse postnatal physiologic conditions, and in disease states such as heart failure. A subset of cardiac-enriched nuclear receptors serve to match mitochondrial fuel preferences and capacity for ATP production with changing energy demands of the heart. In this Review, we describe the role of specific nuclear receptors and their coregulators in the dynamic control of mitochondrial biogenesis and energy metabolism in the normal and diseased heart.
\end{abstract}

\section{Brief overview of cardiac fuel and energy metabolism}

The adult mammalian heart has immense energy demands in order to support its role as a constantly active pump. Indeed, the human heart generates and utilizes kilogram quantities of ATP each day. The vast majority of ATP produced in the cardiac myocyte is generated via oxidative phosphorylation (OXPHOS) within a very-high-capacity mitochondrial system. The heart must continually adapt to changing physiologic conditions that influence workload, as well as alterations in oxygen and energy substrate availability. Notably, given that the heart has a limited capacity for fuel storage, it must utilize several energy substrates in an efficient and dynamic manner. As will be described, members of the nuclear receptor transcription factor superfamily serve to dynamically control preference and capacity for cardiac mitochondrial fuel metabolism during development and in response to myriad physiologic conditions. In addition, alterations in nuclear receptor signaling occur with pathologic cardiac growth and remodeling en route to heart failure.

The adult cardiac myocyte has a higher density of mitochondria than any other cell type (1). The biogenesis of this highcapacity mitochondrial system occurs largely during the perinatal stage of development. This process involves a major burst of mitochondrial biogenesis at birth, followed by a period of mitochondrial maturation and remodeling during the postnatal period (2). Recent evidence indicates that postnatal mitochondrial maturation involves highly coordinated events including mitophagy, biogenesis, and dynamics (fusion and fission), resulting in a mitochondria network that is densely packed between sarcomeres to directly supply ATP to support cardiac contraction (2). The adult

Conflict of interest: D.P. Kelly is a scientific consultant for Pfizer Inc. and receives research support from Takeda Pharmaceutical Co. R.B. Vega received research support from Pfizer Inc.

Reference information: / Clin Invest. 2017;127(4):1155-1164

https://doi.org/10.1172/JCl88888. cardiac mitochondria are equipped with enzymatic machinery capable of oxidizing multiple substrates including fatty acids (the chief fuel) as well as glucose (pyruvate) and ketone bodies. This mitochondrial developmental maturation process occurs in parallel with well-characterized changes in the expression of contractile apparatus isoforms, ion channels, and calcium handling proteins. The collective perinatal programming in energy metabolic and structural genes results in an efficient and enduring pump for the adult life of the organism.

The postnatal heart has an amazing capacity to oxidize multiple fuels and, therefore, is "omnivorous." Pioneering work by multiple groups has defined dynamic shifts in fuel utilization capacity and preferences during development and in disease states. The fetal and immediate postnatal heart relies primarily on glucose (glycolysis) and lactate as energy sources (3-5). However, very soon after birth the heart shifts to fatty acids as the major fuel substrate, coincident with the mitochondrial biogenic response (Figure 1 and refs. 5-8). The level of glucose oxidation also increases (8). The postnatal heart is also capable of oxidizing ketones, albeit at a low level, concomitant with the postnatal rise in circulating ketones (5). The shifts in fuel preference after birth are thought to be driven in part by delivery, which in turn is dependent on hormonal cues and circulating substrate levels such as the rise in circulating fatty acids triggered by the ingestion of milk. However, the capacity for myocardial fatty acid utilization is also driven by gene regulatory events including a major postnatal induction in the expression of genes encoding cellular and mitochondrial fatty acid transporters, as well as mitochondrial fatty acid oxidation (FAO) enzymes (9). In addition, induction of TCA cycle and OXPHOS machinery is necessary to convert the acetyl-CoA product of FAO into ATP. As discussed in the next section, the increase in mitochondrial FAO and oxidative metabolic capacity is orchestrated by a complex transcriptional regulatory circuit involving nuclear receptors that is responsive to physiologic and developmental cues and fuel substrate availability. 


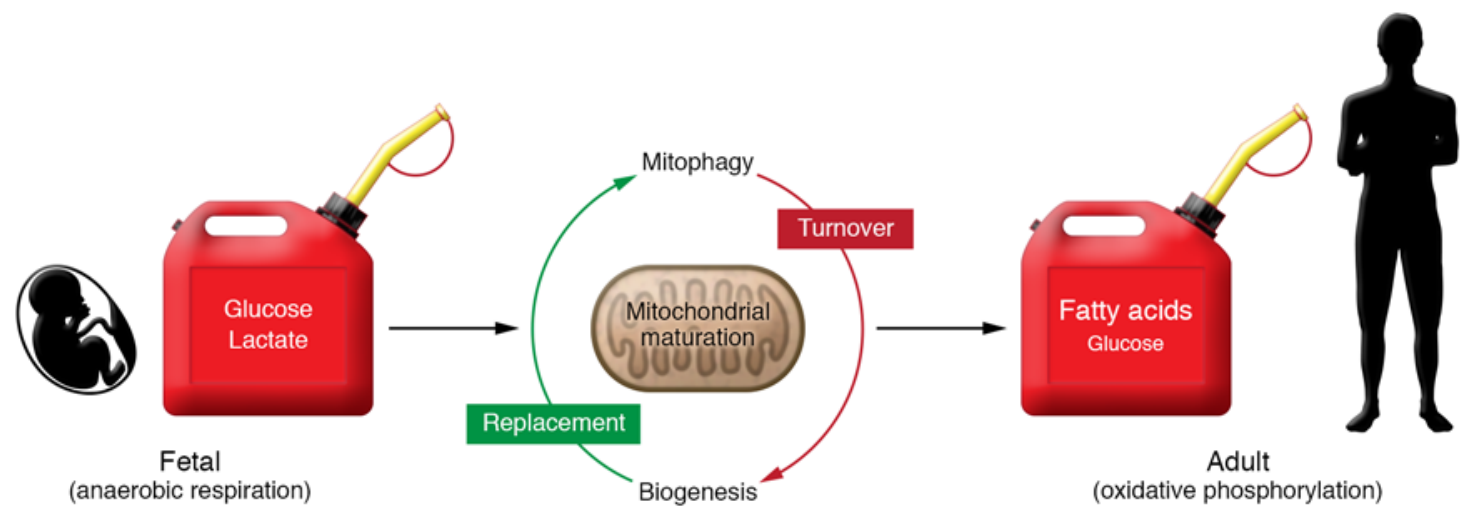

Figure 1. Developmental changes in myocardial fuel substrate preference are linked to mitochondrial biogenesis and maturation. The fetal heart relies primarily on glucose (glycolysis) and lactate as preferred fuel substrates. In the postnatal period FAO becomes the primary energy-producing process. This is preceded by a burst of mitochondrial biogenesis and mitophagy, events that are required to build an adult mitochondria network with high oxidative and ATP synthetic capacity.

\section{Dynamic control of myocardial fuel selection by nuclear receptor signaling}

PPARs match lipid substrate delivery to oxidative capacity. The dramatic increase in the newborn heart's capacity for mitochondrial FAO relies on the ingestion of milk and suggests the existence of a cellular receptor that senses supply and delivery of fatty acid substrate. This provides the heart with the ability to integrate increased substrate delivery with high oxidative capacity. A key breakthrough in establishing this concept was the discovery of the PPARs, members of the nuclear receptor superfamily. PPAR $\alpha$ was originally discovered as a transcriptional regulator of peroxisomal biogenesis and FAO in response to a class of rodent hepatocarcinogens (10). PPAR $\alpha$ and the two other members of this family, PPAR $\delta$ (also known as PPAR $\beta$ ) and PPAR $\gamma$, bind as heterodimers to cognate DNA response elements with the retinoid $\mathrm{X}$ receptor (RXR). All three receptors are expressed in the heart, with PPAR $\alpha$ and PPAR $\delta$ expressed at higher levels than PPAR $\gamma$. Although originally described as a regulator of peroxisomal FAO, PPAR $\alpha$ was subsequently shown to regulate expression of many genes involved in mitochondrial $\beta$-oxidation as well as the fatty acid import machinery (11-15). It is now known that PPAR $\alpha$ gene targets cover all aspects of cellular fatty acid utilization including cellular uptake, storage, and oxidation (16). Genetic loss-of-function studies support these observations. Mice lacking PPAR $\alpha$ have lower cardiac FAO rates and are sensitized to metabolic stress (17-19). Loss of PPAR $\delta$ results in lower FAO rates and cardiac hypertrophy (20). However, there are important differences between PPAR $\alpha$ and PPAR $\delta$. Cardiac-specific overexpression of PPAR $\alpha$ results in high FAO rates and increased triglyceride accumulation $(21,22)$. In contrast, cardiac-specific PPAR $\delta$ transgenes exhibit increased FAO and glucose oxidation rates, without an expansion of myocyteneutral lipid stores (22). Collectively, these results demonstrate that PPAR $\alpha$ and PPAR $\delta$ have overlapping yet distinct gene targets.

PPARs are ligand-activated nuclear receptors with large hydrophobic ligand-binding domains (LBDs). Ligand/receptor binding results in the recruitment of coactivator molecules and stimulation of chromatin remodeling and transcriptional activation (23). The PPAR LBD has been shown to bind a wide range of fatty acids and fatty acid derivatives, with a preference for long-chain unsaturated fatty acids (24-29). Whereas the specific endogenous ligands for PPARs have not been fully characterized, investigations focused on the liver suggest that phosphatidylcholine, derived from de novo lipid synthesis, serves as an endogenous ligand in liver (29). It is likely there are other endogenous ligands, given that phosphatidylcholine binds weakly to PPAR $\delta$ and does not bind PPAR $\gamma$ (29). In the heart, the endogenous PPAR ligands have not been well defined. However, it has been shown that cardiac myocyte triglyceride storage reservoirs are a main source of PPAR $\alpha$ ligands; the Zechner group demonstrated that lipids released from triglycerides by the adipose triglyceride lipase are necessary for full activation of PPAR $\alpha$ (30). In addition, NMRbased studies in mice have shown that PPAR $\alpha$-driven turnover of myocardial triglyceride provides the bulk of substrate for mitochondrial FAO (31). In this way, PPAR $\alpha$ directly senses increased fatty acid delivery, directs storage, and regulates mitochondrial FAO capacity in the adult cardiac myocyte, in order to maintain sufficient ATP generation in accordance with diverse nutritional and physiologic states.

Regulation of cardiac PPAR activity by non-ligand mechanisms. More recently, mechanisms in addition to direct ligand activation have been shown to modulate PPAR $\alpha$ activity in the heart. For instance, sirtuin 1 (Sirt1) has been shown to directly dimerize with PPAR $\alpha$, resulting in displacement of RXR and reduced fatty acid utilization (32). In addition, Krüppel-like factor 15 (KLF15) interacts directly with PPAR $\alpha$ to cooperatively activate FAO gene expression (33). Loss of KLF15 results in reduced cardiac FAO rates and markedly reduced mitochondrial FAO gene expression (34). Another KLF member, KLF5, has been shown to directly regulate PPAR $\alpha$ expression in the heart through binding of the PPAR $\alpha$ promoter (35). Loss of KLF5 in the heart results in lower PPAR $\alpha$ and target gene expression as well as reduced FAO rates. In addition, recent evidence suggests that hypoxia-regulated transcription factors regulate PPAR $\alpha$. The aryl hydrocarbon nuclear translocator (ARNT; also known as HIF1 $\beta$ ) is a basic helix-loop-helix transcription factor that heterodimerizes with HIF1 $\alpha$ and HIF $2 \alpha$ to regulate genes critical for adaptation to oxygen deprivation. Cardiac-specific deletion of Arnt in mice leads to cardiomyopathy with cardiac steatosis (36). This is associated with increased PPAR $\alpha$ expression 
and activation of PPAR $\alpha$ gene targets. An ARNT/HIF2 $\alpha$ heterodimer directly regulates Ppara expression through binding to its promoter (36). Interestingly, HIF1 $\alpha$ has also been shown to activate PPAR $\gamma$ during cardiac hypertrophy and promote lipid accumulation (37). These results underscore the complex regulatory network that exists to regulate PPAR signaling through a variety of physiologic inputs such as $\mathrm{NAD}^{+}$and oxygen availability.

Control of cardiac fuel metabolism by other nuclear receptors. Additional nuclear receptors also likely play an important role in cardiac fuel metabolism. Nuclear receptor 4 subfamily A member 1 (NR4A1), NR4A2, and NR4A3 constitute a family of orphan (ligand unknown) nuclear receptors (38). NR4A1 (also termed Nur77 or NGFIB) regulates glucose metabolism genes in skeletal muscle and other tissues $(39,40)$. Overexpression of NR4A1 in murine muscle results in increased mitochondrial content and activity (41). Notably, NR4A1 expression is acutely and dramatically induced by $\beta$-adrenergic signaling in the heart (42). Interestingly, NR4A1 inhibits isoproterenol-mediated cardiac hypertrophy (42). Given these results, it is possible that cardiac NR4A1 is activated in the heart under stress conditions such as adrenergic stimulation to regulate metabolism as a stress response. However, other studies have demonstrated that NR4A1 exits the nucleus and translocates to the mitochondria to facilitate cytochrome $\mathrm{C}$ release and stimulate apoptosis (43). In support of this model, loss of NR4A1 protects the heart from angiotensin II-induced cardiac hypertrophy (44). Studies to delineate the precise role of NR4A1 signaling in the regulation of cardiac energy metabolism are ongoing.

\section{Control of cardiac mitochondrial biogenesis and dynamics: The estrogen-related receptor/PPAR $\gamma$ coactivator-1 transcriptional regulatory circuit}

The estrogen-related receptor coordinately regulates mitochondrial fuel metabolism and respiratory capacity. In addition to regulating fuel substrate preferences in the adult heart, nuclear receptors serve a key role in the development and remodeling of the cardiac mitochondrial system. As noted above, a burst of mitochondrial biogenesis occurs in heart immediately following birth, including a remarkable increase in the size and number of mitochondria. The estrogen-related receptors (ERRs) serve as critical regulators of this mitochondrial biogenic response. The ERR nuclear receptor family consists of 3 members, ERR $\alpha,-\beta$, and - $\gamma$ encoded by the Esrra, Esrrb, and Esrrg genes, respectively. Although the ERR LBD bears similarity to the estrogen receptor, the ERRs do not bind estrogen and are considered orphan nuclear receptors, as they have no known ligand. $\mathrm{ERR} \alpha$ was originally identified as a regulator of mitochondrial FAO enzymes $(45,46)$. It has now been shown that ERRs regulate target genes in virtually all aspects of mitochondrial energy production, including FAO, OXPHOS, and the electron transport chain (ETC) (47). In the cardiac myocyte, ERR $\alpha$ and ERR $\gamma$ bind directly to response elements in the gene promoters of nuclear-encoded mitochondrial proteins (48). ERR $\alpha$ also directly activates PPAR $\alpha$ expression, providing a feed-forward mechanism to promote fatty acid and oxidative metabolism (47). Interestingly, the transcription factor KLF4 has recently been shown to bind in close proximity to ERR binding sites, including on the Ppara promoter (49). KLF4 synergizes with ERR $\alpha$ to activate target genes and is necessary for normal mitochondrial biogenesis and function in the heart (49).
Loss-of-function studies in mice have highlighted the importance of ERR signaling in the mammalian heart. Esrra-knockout mice are viable and fertile but display reduced cardiac phosphocreatine (PCr) stores, ATP synthesis capacity, and reduced contractile function following pressure overload (50). ERR $\gamma$ is also necessary for normal cardiac function, as genetic deletion results in perinatal lethality with a failure to transition to oxidative metabolism in heart (51). Finally, postnatal and cardiac-specific deletion of Esrrg on an Esrra-null background results in a lethal cardiomyopathy characterized by abnormalities in mitochondrial structure and function and reduced energy production capacity (52). These results underscore the central role of ERRs in the broad control of cardiac mitochondrial function and also highlight the functional overlap and redundancy of ERR $\alpha$ and $-\gamma$ in the mammalian heart. Esrrb knockout mice die at embryonic day 10.5 due to severe placental defects (53). The role of ERR $\beta$ in adult heart has not been fully evaluated. Inducible cardiac-specific loss-of-function models will aid in determining a role for ERR $\beta$ in the heart.

Interestingly, in addition to classic mitochondrial metabolic targets, ERRs also bind directly to response elements within the regulatory regions of the promoters of contractile, structural and calcium-handling genes (48). Overexpression studies show that ERR $\alpha$ and $-\gamma$ can activate the expression of a number of contractile and calcium-handling genes in cardiac myocytes, including Myh6 ( $\alpha \mathrm{MHC}$ ) and Atp2a2 (SERCA2A) $(47,48)$. A similar mechanism is operative in skeletal muscle where an ERR $\gamma$ miRNA circuit works to directly regulate $M y h 7$ ( $\beta \mathrm{MHC} / \mathrm{MHC}-\mathrm{I})$ and the slow, type I fiber program (54). Finally, ERR $\gamma$ has been shown to improve the efficiency of reprogramming fibroblasts into cardiac myocytes (55). Taken together, these results indicate that ERR signaling coordinately regulates energy metabolic and structural programs in striated muscle and may be critical in matching ATP production with contractile function.

Integration of upstream stimuli with the nuclear receptor-mediated control of mitochondrial biogenesis and dynamics: the PPAR $\gamma$ coactivator-1 coactivators. A complex array of extracellular and developmental stimuli must be received and transmitted to coordinate mitochondrial biogenesis and function during heart development and in various physiologic contexts. A key to our understanding of how the myocyte transduces these inputs came with the discovery of PPAR $\gamma$ coactivator- $1 \alpha$ (PGC-1 $\alpha$, encoded by Ppargc1a). PGC- $1 \alpha$ was originally discovered as a cold-inducible transcriptional coactivator of PPAR $\gamma$ in brown adipose tissue (56). PGC-1 $\alpha$ is a member of a family of transcriptional coregulators that include PGC- $1 \beta$ and the more distant PGC-1-related coactivator (57-59). PGC-1 $\alpha$ interacts with and coactivates a variety of nuclear receptors, including PPARs and ERRs, via distinct LXXLL motifs. Interestingly, PGC- $1 \alpha$ expression is rapidly induced by a number of stimuli, such as cold exposure, $\beta$-adrenergic stimulation, and exercise (Figure 2 and refs. 60-62). The effects on PGC- $1 \alpha$ from these physiologic stimuli are transduced by numerous intracellular signaling pathways and effectors including AMPK, calmodulin-dependent kinase, calcineurin, and Sirt1 (Figure 2 and ref. 9). PGC- $1 \alpha$ expression is also regulated during development and is induced following birth, indicating that this coregulator plays a central role in the postnatal mitochondrial biogenic response (63). The highly inducible nature of PGC-1 $\alpha$ expression provides a layer of regula- 


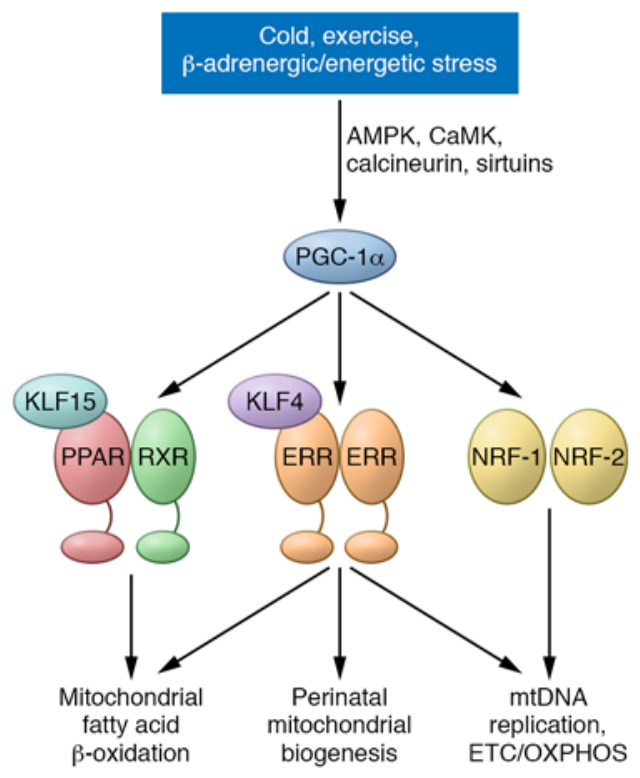

Figure 2. Cardiac nuclear receptors respond to physiologic cues transduced by the inducible transcriptional coactivator PGC-1 $\alpha$. Various physiologic stimuli increase cardiac output and energy requirements of the heart. These signals are transmitted through a variety of cellular signaling pathways that regulate PGC- $1 \alpha$ expression and/or activity. PGC- $1 \alpha$ interacts directly with multiple nuclear receptors and other transcription factors to orchestrate mitochondrial biogenesis and function, including increasing capacity for oxidizing fatty acids. mtDNA, mitochondrial DNA.

tion that integrates extracellular signals with downstream effectors, i.e., nuclear receptors (Figure 2). This is likely of particular importance in the control of orphan receptors that may lack a true endogenous activating ligand such as ERRs. The interactions of PGC- $1 \alpha$ are not limited to nuclear receptors. For instance, PGC- $1 \alpha$ also coactivates the nuclear respiratory factors NRF-1 and NRF-2 $(60,64)$. NRF-1 and -2 activate expression of multiple respiratory chain subunits in addition to the mitochondrial transcription factor TFAM (65-67). This latter circuit provides one mechanism for the coordinate control of nuclear and mitochondrial genomes. Thus, PGC-1 functions as the "conductor" of a coordinated mitochondrial gene regulatory program (Figure 2).

PGC- 1 transgenic and gene deletion studies in mice have provided significant insight into the role of these coregulators in the development of the high-capacity mitochondrial system in heart $(63,68-71)$. Such studies demonstrate that PGC- $1 \alpha$ is necessary for maximal ATP synthesis and FAO, likely working largely with the ERRs (72). Deletion of the genes encoding PGC- $1 \alpha$ or PGC-1 $\beta$ separately in mice results in a minimal phenotype at baseline (69) but causes modest cardiac dysfunction following pressure overload $(73,74)$. However, mice lacking both PGC- $1 \alpha$ and $-1 \beta$ die shortly after birth due to cardiomyopathy caused by an arrest in perinatal mitochondrial biogenesis (70). In addition, conditional disruption of the Ppargc1a/b in the immediate postnatal period after the burst of biogenesis results in a defect in normal mitochondrial maturation that leads to a progressive, lethal cardiomyopathy due to alterations in mitochondrial fusion and fission (71). Interestingly, expression of several factors critical for mitochondrial fusion and fission, including mitofusin 2 (MFN2) and MFN1, is reduced in the Ppargc1a/b knockout (71). Consistent with this finding, both MFN1 and MFN2 have been identified as direct ERR $\alpha /$ PGC-1 $\alpha$ gene targets $(71,75)$.

Surprisingly, cardiac-specific, inducible deletion of Ppargc1b on a systemic Ppargc1a-null background in adult mice results in a rather minimal mitochondrial structural phenotype without overt cardiac dysfunction. However, the hearts of these mice exhibit a marked and global reduction in expression of nuclear-encoded mitochondrial genes and reduced State 3 respiration $(71,76)$. Compared with the neonatal phenotype, the minimal cardiac phenotype of adult PGC- $1 \alpha / \beta$ deficiency may reflect relatively low rates of mitochondrial turnover and replacement (mitophagy) in the normal adult heart. It is also possible compensatory factors are activated in the adult heart in the context of PGC $-1 \alpha / \beta$ deficiency.

Turnover of damaged or old mitochondria in the cell involves a process termed "mitophagy," linked to a culling process that involves fission and fusion (77). Mitophagy is controlled and regulated by the PTEN-induced kinase 1/Parkin (PINK1/Parkin) pathway. PINK1 is recruited to mitochondria but normally undergoes rapid proteolytic degradation. However, damage to mitochondria resulting in membrane depolarization stabilizes PINK1, which in turn phosphorylates MFN2 and other proteins that serve as targets for the E3 ubiquitin ligase, Parkin. Parkin-mediated ubiquitination of outer mitochondrial membrane proteins targets the mitochondria for engulfment by the autophagosome. In support of this model, loss of MFN2 prevents Parkin from docking to depolarized mitochondria $(78,79)$. Therefore, inhibition of Parkin-MFN2 docking or MFN2 phosphorylation will block mitophagy. Indeed, cardiac expression of a non-phosphorylatable MFN2 mutant (MFN2AA) prevents mitochondrial recruitment of Parkin and results in reduced mitochondrial respiratory capacity and ultrastructural mitochondrial defects in mice (80). Specifically, MFN2AA mice develop cardiomyopathy and die prematurely by 8 weeks of age (80). Interestingly, the resultant cardiac mitochondria maintain a fetal phenotype similar to that seen with PGC- $1 \alpha / \beta$ deficiency (80). In turn, postnatal induction of ERR and PPAR $\alpha$ target gene expression is also blocked in these mice. Given that the MFN2AA mouse phenocopies perinatal PGC- $1 \alpha / \beta$ deficiency, these results strongly suggest that mitophagy of fetal mitochondria precedes replacement (biogenesis). Together these results suggest that the transition to adult cardiac metabolism requires the replacement of fetal with adult mitochondria through a coordinated mitophagy and biogenic circuit that involves regulatory cross-talk between the mitophagy machinery and the PGC-1/ nuclear receptor circuit (Figure 1).

The coordinated action of PPAR, ERR, and PGC-1 provides a circuitry for postnatal energy metabolic maturation of the mammalian heart. The supply of fatty acid-rich milk after birth provides the signal to PPAR for a switch in fuel preference to oxidative metabolism. This is reliant on a mitochondrial network with the capacity to support high rates of oxidative metabolism. Through a number of loss-of-function studies in mice, described in the preceding sections, it is apparent that PGC-1 and ERR signaling orchestrate this mitochondrial biogenic response. In addition, recent evidence strongly suggests that the biogenic response is coordinately regulated with mitophagic removal of fetal mitochondria and replacement with mature adult mitochondria. Furthermore, this system must also have regulatory cross-talk with programs that regulate 


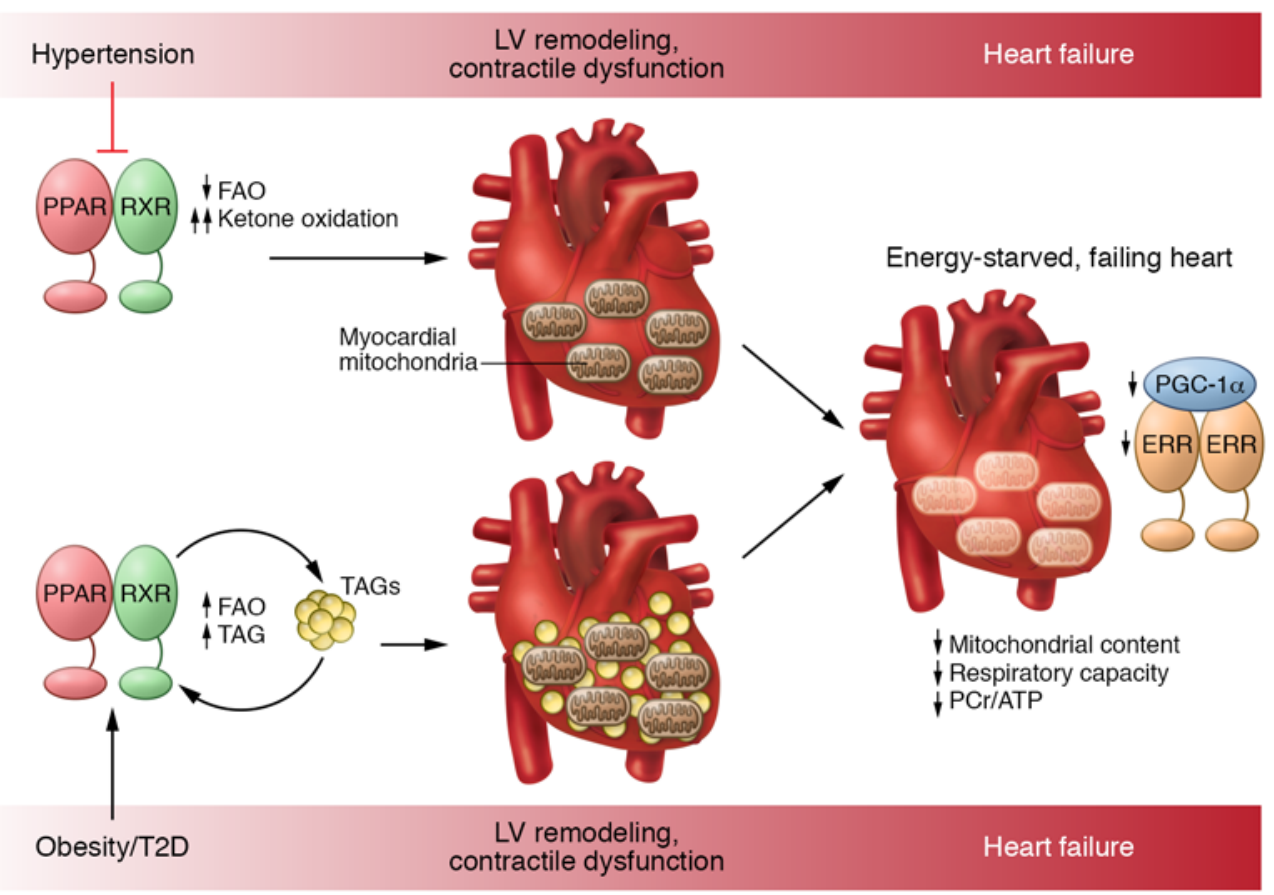

Figure 3. Disease etiology-specific perturbations in nuclear receptor signaling result in fuel and energy metabolism derangements in the failing heart. Deactivation of nuclear receptor signaling contributes to derangements of fuel substrate utilization and mitochondrial energy metabolism during the progression of hypertensive heart disease (the energy-starved heart). Early in the disease process, PPAR signaling becomes inhibited, resulting in a decreased reliance on fatty acids as the preferred fuel substrate. A different phenotype is observed in the setting of diabetes and obesity (the overfed lipotoxic heart). PPAR signaling becomes chronically activated, resulting in increased rates of FAO and exuberant neutral lipid storage. Later in the disease process, a common pathogenic pathway of diminished ERR and PCC-1 signaling leads to decreased mitochondrial energy production and content, resulting in an energy-starved failing heart. This condition manifests as decreased PCr/ATP ratios and impaired contractile efficiency. T2D, type 2 diabetes.

energy utilization, such as sarcomeric, ion channel, and calcium handling gene expression, to match ATP production with demands. Future investigation is needed to more clearly delineate the role of this regulatory network during cardiac myocyte maturation.

\section{Energy metabolic derangements in the failing heart}

Altered nuclear receptor signaling contributes to fuel metabolic derangements in the hypertrophied and failing heart. During the development of pathologic cardiac hypertrophy due to pressure overload or myocardial ischemic insult, such as occurs in hypertensive heart disease, the heart undergoes a reactivation of the so-called "fetal gene program." This process involves induction of contractile, structural, and calcium handling genes that are normally highly expressed in the developing fetal heart and skeletal muscle. For instance, the $M y h 7$ gene ( $\beta-\mathrm{MHC})$ is activated while the adult isoform, Myh6 ( $\alpha$-MHC), is repressed (81). Interestingly, a similar pattern is observed with fuel metabolism in the failing heart. Myocardial FAO rates decline early on in pathologic cardiac hypertrophic growth, concordant with an increase in glucose utilization, a fetal pattern of substrate utilization (Figure 3 and refs. 82-87). More recently it has been demonstrated that ketones are also used as an alternative fuel in the failing heart, possibly to compensate for the reduced capacity to burn fatty acids $(88,89)$.

Multiple lines of evidence in animal models and in humans support the conclusion that the reduced capacity for fatty acid utilization in the hypertrophied and failing heart involves gene regulatory mechanisms related to the deactivation of PPAR $\alpha$ signaling (Figure 3 and refs. 32, 90, 91). The deactivation of PPAR $\alpha$ signaling in the hypertrophied and failing heart occurs during the early stages and at both transcriptional and posttranscriptional levels $(90,92)$. Well-known effectors of cardiac hypertrophy and remodeling, such as angiotensin II and hypoxia, reduce activity of PPAR $\alpha$, leading to a reduction in the rate of mitochondrial FAO (93-95). In addition, decreased generation of activating ligands through reduced triglyceride droplet dynamics may also contribute to decreased PPAR $\alpha$ signaling in the failing heart (96). The role of altered PPAR signaling and fuel shifts as causative in the pathogenesis of cardiac functional and structural remodeling (versus as a secondary phenomenon) is an area of active investigation. In support of the former, a recent interesting study from the Tian laboratory demonstrated that mice genetically engineered with persistently high myocardial FAO rates are relatively resistant to pressure overload-induced pathologic remodeling (97).

A very different fuel metabolic shift occurs with cardiac dysfunction in the setting of metabolic syndrome and type 2 diabetes. Multiple studies have demonstrated an increase in myocardial FAO and triglyceride content in humans with diabetes and metabolic syndrome (98-100). In some studies, myocardial steatosis has been shown to be an independent predictor of pathologic remodeling and cardiac dysfunction (101-103). Interestingly, the increased lipid accumulation is associated with higher myocardial FAO rates in diabetic patients, at least in the early stages of disease $(104,105)$. Given the known relationship between myocellular lip- 
id dynamics and the control of mitochondrial FAO, it was reasonable to hypothesize that PPAR $\alpha$ is involved in the pathogenesis of diabetic cardiac dysfunction. In support of this notion, cardiac-specific overexpression of PPAR $\alpha$ in mice (MHC-PPAR $\alpha$ mice) exhibits many signatures of diabetic cardiomyopathy, including high FAO, myocyte lipid accumulation, and ventricular remodeling (21). These effects are worsened when the mice are placed on a high-fat diet (106). In addition, MHC-PPAR $\alpha$ mice are sensitized to myocardial ischemic insult (22) such as occurs in the diabetic state. The expression of many genes involved in fatty acid uptake and storage is also increased in these mice (21). These results support a model wherein increased delivery of fatty acids in the context of myocardial insulin resistance leads to chronic activation of PPAR $\alpha$ signaling. This chronic activation results in high rates of flux through the mitochondrial FAO pathway and progressive accumulation of neutral lipid stores, leading to diabetic "lipotoxic cardiomyopathy" (Figure 3). Consistent with this model, NMR studies have shown a dramatic increase in myocardial triglyceride turnover in mouse models of type 2 diabetes, including the MHC-PPAR $\alpha$ model (31).

Interestingly, cardiac-specific overexpression of the related nuclear receptor, PPAR $\delta$ (MHC-PPAR $\delta$ mice), which activates many of the same FAO gene targets as PPAR $\alpha$, does not cause neutral lipid accumulation or cardiomyopathy in mice (22). As predicted by the lack of a lipid accumulation phenotype in MHC-PPAR $\delta$ mice, many of the PPAR $\alpha$ target genes involved in fatty acid uptake and storage are not activated by PPAR $\delta$. Additionally, expression of angiopoietin-like 4, an endogenous inhibitor of lipoprotein lipase, is activated by $\operatorname{PPAR} \delta$ (107). Similar to the cardiac phenotype of PPAR $\alpha$ transgenic mice, cardiac overexpression of PPAR $\gamma$ also leads to dilated cardiomyopathy with increased FAO rates and lipid accumulation (108). Unexpectedly, deletion of PPAR $\alpha$ rescued this phenotype (109). This latter observation suggests that "cross-talk" occurs between PPAR $\alpha$ and PPAR $\gamma$ in the hearts of these animals. It should be noted that despite the observation of the close association between myocyte lipid accumulation and cardiac dysfunction, the fundamental cellular basis for lipotoxic cardiomyopathy remains unknown. Indeed it is possible that the lipid accumulation actually serves a protective function.

There is extensive clinical experience with pharmacologic PPAR $\alpha$ ligands, through the use of lipid-lowering fibrates. However, the impact of PPAR $\alpha$ ligands on cardiovascular outcomes, based on the results of trials in humans, is mixed, possibly reflecting distinct effects on dyslipidemia (vascular disease) and cardiac function (110). It should be noted that the effect of PPAR $\alpha$ agonists on myocardial function and substrate utilization in humans is largely unknown. Interestingly, one small study in healthy volunteers demonstrated no effect on fatty acid utilization or oxidation with fenofibrate (111). A primary target of fibrates is the liver, and in this study treatment with fenofibrate resulted in reduced secretion of triglyceride. Therefore, fibrates may decrease lipid substrate and activate the delivery of ligands to the heart. If this is the case, then cardiac PPAR $\alpha$ signaling might also be decreased in some circumstances.

Pathologic derangements in the PGC-1/ERR circuit: A final common pathway to the failing, "energy-starved heart." Following disease-specific changes in fuel preference during early-stage pathologic cardiac remodeling, significant evidence supports the notion that the later-stage failing heart, regardless of etiology, develops mitochondrial dysfunction and diminished capacity for ATP production. Indeed, a diminished ratio of PCr to ATP has been shown to be an excellent prognostic indicator of survival in human heart failure $(112,113)$. Gene expression and proteomic profiling, together with ultrastructural studies, indicate that the expression of genes and proteins involved in mitochondrial energy transduction and respiration is downregulated in the late-stage failing heart (114-117). In addition, several recent studies have revealed changes in the metabolome indicative of alterations (bottlenecks) in fuel oxidation and mitochondrial respiration $(87,89,117)$. These observations have led to a description of the failing heart as energy starved, a condition that can result from any of a myriad of potential metabolic derangements that occur during heart failure, ranging from alterations in fuel oxidation to ATP synthesis and storage. The key question remains as to whether these alterations in energy metabolism are contributory or merely a consequence of heart failure. As described herein, increasing evidence supports the former conclusion.

Several lines of evidence support the conclusion that altered activity of ERR and PGC-1 contribute to mitochondrial dysfunction in the failing heart. First, genetic deletion of Esrra in mice results in impaired capacity of mitochondria to produce ATP and also in stress-induced cardiac dysfunction (50). Secondly, Ppargc1aknockout mice develop accelerated heart failure following pressure overload (73). In addition, although cardiac-specific inactivation of both PGC- $1 \alpha$ and $-1 \beta$ in the adult heart does not lead to overt dysfunction, it does produce abnormalities in phospholipid biosynthesis that may impair mitochondrial function. Mice lacking both cardiac PGC- $1 \alpha$ and $-1 \beta$ contain mitochondria that display a phenotype reminiscent of mitochondria found in patients with Barth syndrome, a rare genetic disorder caused by the inability to produce mature cardiolipin (76). Indeed, Ppargc1a/b-knockout mice have reduced cardiolipin and impaired mitochondrial respiration. Third, a number of studies in animal models and in humans have shown reduced levels of PGC-1 $\alpha$ and ERR transcripts and protein levels in heart failure (117-121). Collectively, these data clearly connect diminished mitochondrial respiration capacity with reduced ERR/PGC-1 signaling and suggest that this may be a final common pathway in heart failure (Figure 3).

Recent studies also suggest that loss of PGC- $1 \alpha$ is associated with peripartum cardiomyopathy (PPCM). PPCM is a common disorder that occurs in the last month of pregnancy or in the period immediately following birth. Although the precise cause is unknown, some evidence implicates hormonal imbalances that primarily affect the vasculature in the heart (122). Interestingly, cardiac-specific Ppargcla-knockout mice develop PPCM (123). Loss of PGC-1 $\alpha$ results in impaired angiogenesis and reduced VEGFA expression (123). These alterations, combined with strong antiangiogenic signaling during pregnancy, are believed to be a trigger for PPCM.

Active repression of nuclear receptor signaling may also contribute to impaired mitochondrial energetics en route to heart failure. Chicken OVA upstream promoter (COUP) transcription factor 2 (COUP-TFII, also known as NR2F2) is an orphan nuclear receptor that is expressed at low levels in the heart but that increases in pathologic hypertrophy and heart failure $(124,125)$. Cardiac-specific overexpression of COUP-TFII in mice results in cardiomyop- 
athy and premature death (125). Remarkably, these mice display marked downregulation of expression of mitochondrial energy metabolic genes including FAO and the ETC. These alterations appear to be due to direct inhibition of PPAR $\alpha, E R R / \gamma$, and PGC- $1 \alpha$ by COUP-TFII (125). Additionally, other proteins may directly inhibit coactivation of PPARs and ERRs by PGC- $1 \alpha$. The corepressor molecule receptor-interacting protein 140 (RIP140) interacts with nuclear receptors in a ligand-dependent manner (126). RIP140 is expressed in many tissues with a high metabolic rate, including the heart. Its expression is increased in experimental heart failure and inhibits PGC- $1 \alpha$ target gene expression (127). Cardiac overexpression of RIP140 results in cardiomyopathy and premature death (128). Furthermore, cardiac RIP140 transgenic mice have markedly suppressed mitochondrial respiration with decreased expression of ERR and PPAR targets (128). These results imply that there is a delicate balance between activating and repressing transcription factors/coregulators in the heart to regulate energy production. Perturbations in this circuit may contribute to the energy metabolic derangements known to occur in heart failure. These mechanisms should be considered as therapeutic targets aimed at improving mitochondrial energy production and function.

A key question for the field relates to whether modulation of PPAR, ERR, or PGC-1 activity can reverse or retard the development of heart failure. Given that the fuel metabolic abnormalities occur early in the disease process and are etiology specific (Figure 3), PPAR signaling or attendant downstream pathways would appear to be attractive targets. However, proof of concept is largely lacking for the reversal of the fuel metabolic disturbances in either the energy-starved hypertensive heart or the "overfed" diabetic heart. Moreover, diabetes and hypertension often co-exist in heart failure patients, such that metabolic biomarkers would be needed in order to define the dominant process. Later stages of cardiac remodeling may be amenable to strategies aimed at reactivating ERR and/or PGC-1.

\section{Conclusions and perspectives}

Nuclear receptors in the heart play a fundamental role in the control of energy metabolism and homeostasis. PPARs and ERRs serve as critical regulators and sensors for fuel substrate utilization and mitochondrial biogenesis. Upstream physiologic, hormonal, and developmental cues are integrated with the actions of PPARs and ERRs through the PGC-1 transcriptional coactivators. These factors work in concert during the postnatal period as the heart undergoes a massive burst of mitochondrial biogenesis to establish a high capacity for ATP production. Recent evidence links this transcriptional regulatory circuitry to the mitochondrial mitophagy machinery. Significant evidence indicates that nuclear receptor signaling becomes dysregulated during pathologic hypertrophy and in the failing heart. In many ways, deactivation of PPARs, ERRs, and/or PGC-1 in the hypertrophied and failing heart, due to pressure overload, mimics the fetal condition with a shift back toward glycolysis, a more primitive fuel pathway. In contrast, the diabetic heart shifts to high utilization of fatty acid substrates. Proof-of-concept studies are underway to rigorously assess the potential for targeting cardiac nuclear receptors as a metabolic modulatory strategy aimed at the relatively early stages of heart failure.

\section{Acknowledgments}

We wish to thank Teresa C. Leone for critical reading and Lorenzo Thomas for assistance with manuscript preparation. This work was supported by NIH grants R01 DK045416 (to DPK), R01 HL058493 (to DPK), and R01 HL128349 (to DPK).

Address correspondence to: Daniel P. Kelly, Sanford Burnham Prebys Medical Discovery Institute at Lake Nona, 6400 Sanger Road, Orlando, Florida 32827, USA. Phone: 407.745.2096; E-mail: dkelly@sbpdiscovery.org.
1. Goffart S, von Kleist-Retzow JC, Wiesner RJ. Regulation of mitochondrial proliferation in the heart: power-plant failure contributes to cardiac failure in hypertrophy. Cardiovasc Res. 2004;64(2):198-207.

2. Dorn GW, Vega RB, Kelly DP. Mitochondrial biogenesis and dynamics in the developing and diseased heart. Genes Dev. 2015;29(19):1981-1991.

3. Lopaschuk GD, Spafford MA. Energy substrate utilization by isolated working hearts from newborn rabbits. Am J Physiol. 1990; 258(5 pt 2):H1274-H1280.

4. Lopaschuk GD, Spafford MA, Marsh DR. Glycolysis is predominant source of myocardial ATP production immediately after birth. Am J Physiol. 1991;261(6 pt 2):H1698-H1705.

5. Bartelds B, et al. Perinatal changes in myocardial supply and flux of fatty acids, carbohydrates, and ketone bodies in lambs. Am J Physiol. 1998; 274(6 pt 2):H1962-H1969.

6. Bing RJ, Siegel A, Ungar I, Gilbert M. Metabolism of the human heart. II. Studies on fat, ketone and amino acid metabolism. Am JMed. 1954;16(4):504-515.

7. Werner JC, Sicard RE. Lactate metabolism of isolated, perfused fetal, and newborn pig hearts.
Pediatr Res. 1987;22(5):552-556.

8. Itoi T, Lopaschuk GD. The contribution of glycolysis, glucose oxidation, lactate oxidation, and fatty acid oxidation to ATP production in isolated biventricular working hearts from 2-week-old rabbits. Pediatr Res. 1993;34(6):735-741.

9. Vega RB, Horton JL, Kelly DP. Maintaining ancient organelles: mitochondrial biogenesis and maturation. Circ Res. 2015;116(11):1820-1834.

10. Issemann I, Green S. Activation of a member of the steroid hormone receptor superfamily by peroxisome proliferators. Nature. 1990;347(6294):645-650.

11. Gulick T, Cresci S, Caira T, Moore DD, Kelly DP. The peroxisome proliferator-activated receptor regulates mitochondrial fatty acid oxidative enzyme gene expression. Proc Natl Acad Sci U S A. 1994;91(23):11012-11016.

12. Brandt JM, Djouadi F, Kelly DP. Fatty acids activate transcription of the muscle carnitine palmitoyltransferase I gene in cardiac myocytes via the peroxisome proliferator-activated receptor alpha. J Biol Chem. 1998;273(37):23786-23792.

13. Mascaró C, Acosta E, Ortiz JA, Marrero PF, Hegardt FG, Haro D. Control of human muscle-type carnitine palmitoyltransferase I gene transcription by peroxisome proliferator-activated receptor. J Biol Chem. 1998;273(15):8560-8563.

14. van der Meer DL, et al. Profiling of promoter occupancy by PPARalpha in human hepatoma cells via ChIP-chip analysis. Nucleic Acids Res. 2010;38(9):2839-2850.

15. McMullen PD, et al. A map of the PPAR $\alpha$ transcription regulatory network for primary human hepatocytes. Chem Biol Interact. 2014;209:14-24.

16. Kersten S. Integrated physiology and systems biology of PPAR $\alpha$. Mol Metab. 2014;3(4):354-371.

17. Djouadi F, Brandt JM, Weinheimer CJ, Leone TC, Gonzalez FJ, Kelly DP. The role of the peroxisome proliferator-activated receptor alpha $(\operatorname{PPAR} \alpha)$ in the control of cardiac lipid metabolism. Prostaglandins Leukot Essent Fatty Acids. 1999;60(5-6):339-343.

18. Leone TC, Weinheimer CJ, Kelly DP. A critical role for the peroxisome proliferator-activated receptor alpha $(\mathrm{PPAR} \alpha)$ in the cellular fasting response: the PPAR $\alpha$-null mouse as a model of fatty acid oxidation disorders. Proc Natl Acad Sci US A. 1999;96(13):7473-7478.

19. Watanabe K, et al. Constitutive regulation of cardiac fatty acid metabolism through peroxisome proliferator-activated receptor alpha associated 
with age-dependent cardiac toxicity. J Biol Chem. 2000;275(29):22293-22299.

20. Wang P, et al. Peroxisome proliferator-activated receptor $\{$ delta\} is an essential transcriptional regulator for mitochondrial protection and biogenesis in adult heart. Circ Res. 2010;106(5):911-919.

21. Finck BN, et al. The cardiac phenotype induced by PPAR $\alpha$ overexpression mimics that caused by diabetes mellitus. JClin Invest. 2002;109(1):121-130.

22. Burkart EM, et al. Nuclear receptors PPAR $\beta / \Delta$ and PPAR $\alpha$ direct distinct metabolic regulatory programs in the mouse heart. J Clin Invest. 2007;117(12):3930-3939.

23. Rastinejad F, Huang P, Chandra V, Khorasanizadeh S. Understanding nuclear receptor form and function using structural biology. J Mol Endocrinol. 2013;51(3):T1-T21.

24. Göttlicher M, Widmark E, Li Q, Gustafsson JA. Fatty acids activate a chimera of the clofibric acid-activated receptor and the glucocorticoid receptor. Proc Natl Acad Sci U S A. 1992;89(10):4653-4657.

25. Keller H, Dreyer C, Medin J, Mahfoudi A, Ozato $\mathrm{K}$, Wahli W. Fatty acids and retinoids control lipid metabolism through activation of peroxisome proliferator-activated receptor-retinoid $\mathrm{X}$ receptor heterodimers. Proc Natl Acad Sci U S A. 1993;90(6):2160-2164.

26. Forman BM, Tontonoz P, Chen J, Brun RP, Spiegelman BM, Evans RM. 15-Deoxy- $\Delta$ 12, 14-prostaglandin $\mathrm{J} 2$ is a ligand for the adipocyte determination factor PPAR $\gamma$. Cell. 1995;83(5):803-812.

27. Kliewer SA, et al. Fatty acids and eicosanoids regulate gene expression through direct interactions with peroxisome proliferator-activated receptors $\alpha$ and $\gamma$. Proc Natl Acad Sci U S A. 1997;94(9):4318-4323.

28. Xu HE, et al. Molecular recognition of fatty acids by peroxisome proliferator-activated receptors. Mol Cell. 1999;3(3):397-403.

29. Chakravarthy MV, et al. Identification of a physiologically relevant endogenous ligand for PPAR $\alpha$ in liver. Cell. 2009;138(3):476-488.

30. Haemmerle G, et al. ATGL-mediated fat catabolism regulates cardiac mitochondrial function via PPAR- $\alpha$ and PGC-1. Nat Med. 2011;17(9):1076-1085.

31. Banke NH, et al. Preferential oxidation of triacylglyceride-derived fatty acids in heart is augmented by the nuclear receptor PPARalpha. Circ Res. 2010;107(2):233-241.

32. Oka S, et al. Peroxisome proliferator activated receptor- $\alpha$ association with silent information regulator 1 suppresses cardiac fatty acid metabolism in the failing heart. Circ Heart Fail. 2015;8(6):1123-1132.

33. Prosdocimo DA, et al. KLF15 and PPAR $\alpha$ cooperate to regulate cardiomyocyte lipid gene expression and oxidation. PPAR Res. 2015;2015:201625.

34. Prosdocimo DA, et al. Kruppel-like factor 15 is a critical regulator of cardiac lipid metabolism. J Biol Chem. 2014;289(9):5914-5924.

35. Drosatos K, et al. Cardiac myocyte KLF5 regulates Ppara expression and cardiac function. Circ Res. 2016;118(2):241-253.

36. Wu R, et al. Cardiac-specific ablation of ARNT leads to lipotoxicity and cardiomyopathy. JClin
Invest. 2014;124(11):4795-4806.

37. Krishnan J, et al. Activation of a HIF1 $\alpha-P P A R \gamma$ axis underlies the integration of glycolytic and lipid anabolic pathways in pathologic cardiac hypertrophy. Cell Metab. 2009;9(6):512-524.

38. Pearen MA, Muscat GE. Minireview: Nuclear hormone receptor $4 \mathrm{~A}$ signaling: implications for metabolic disease. Mol Endocrinol. 2010;24(10):1891-1903.

39. Chao LC, Zhang Z, Pei L, Saito T, Tontonoz P, Pilch PF. Nur77 coordinately regulates expression of genes linked to glucose metabolism in skeletal muscle. Mol Endocrinol. 2007;21(9):2152-2163.

40. Close AF, Rouillard C, Buteau J. NR4A orphan nuclear receptors in glucose homeostasis: a minireview. Diabetes Metab. 2013;39(6):478-484.

41. Chao LC, et al. Skeletal muscle Nur77 expression enhances oxidative metabolism and substrate utilization. J Lipid Res. 2012;53(12):2610-2619.

42. Yan G, et al. Orphan nuclear receptor Nur77 inhibits cardiac hypertrophic response to $\beta$-adrenergic stimulation. Mol Cell Biol. 2015;35(19):3312-3323.

43. Cheng Z, et al. Mitochondrial translocation of Nur77 mediates cardiomyocyte apoptosis. Eur Heart J. 2011;32(17):2179-2188.

44. Wang RH, et al. The orphan receptor TR3 participates in angiotensin II-induced cardiac hypertrophy by controlling mTOR signalling. EMBO Mol Med. 2013;5(1):137-148.

45. Vega RB, Kelly DP. A role for estrogen-related receptor alpha in the control of mitochondrial fatty acid $\beta$-oxidation during brown adipocyte differentiation. J Biol Chem. 1997;272(50):31693-31699.

46. Sladek R, Bader JA, Giguère V. The orphan nuclear receptor estrogen-related receptor $\alpha$ is a transcriptional regulator of the human medium-chain acyl coenzyme A dehydrogenase gene. Mol Cell Biol. 1997;17(9):5400-5409.

47. Huss JM, Torra IP, Staels B, Giguère V, Kelly DP. Estrogen-related receptor $\alpha$ directs peroxisome proliferator-activated receptor alpha signaling in the transcriptional control of energy metabolism in cardiac and skeletal muscle. Mol Cell Biol. 2004;24(20):9079-9091.

48. Dufour CR, et al. Genome-wide orchestration of cardiac functions by the orphan nuclear receptors ERR $\alpha$ and $\gamma$. Cell Metab. 2007;5(5):345-356.

49. Liao X, et al. Kruppel-like factor 4 is critical for transcriptional control of cardiac mitochondrial homeostasis. JClin Invest. 2015;125(9):3461-3476.

50. Huss JM, et al. The nuclear receptor ERR $\alpha$ is required for the bioenergetic and functional adaptation to cardiac pressure overload. Cell Metab. 2007;6(1):25-37.

51. Alaynick WA, et al. ERR $\gamma$ directs and maintains the transition to oxidative metabolism in the postnatal heart. Cell Metab. 2007;6(1):13-24.

52. Wang T, et al. Estrogen-related receptor $\alpha$ (ERR $\alpha$ ) and ERR $\gamma$ are essential coordinators of cardiac metabolism and function. Mol Cell Biol. 2015;35(7):1281-1298.

53. Luo J, Sladek R, Bader JA, Matthyssen A, Rossant J, Giguère V. Placental abnormalities in mouse embryos lacking the orphan nuclear receptor ERR-ß. Nature. 1997;388(6644):778-782.

54. Gan Z, et al. Nuclear receptor/microRNA circuitry links muscle fiber type to energy metabolism.
JClin Invest. 2013;123(6):2564-2575.

55 . Fu JD, et al. Direct reprogramming of human fibroblasts toward a cardiomyocyte-like state. Stem Cell Reports. 2013;1(3):235-247.

56. Puigserver P, Wu Z, Park CW, Graves R, Wright $\mathrm{M}$, Spiegelman BM. A cold-inducible coactivator of nuclear receptors linked to adaptive thermogenesis. Cell. 1998;92(6):829-839.

57. Andersson U, Scarpulla RC. Pgc-1-related coactivator, a novel, serum-inducible coactivator of nuclear respiratory factor 1-dependent transcription in mammalian cells. Mol Cell Biol. 2001;21(11):3738-3749.

58. Lin J, Puigserver P, Donovan J, Tarr P, Spiegelman BM. Peroxisome proliferator-activated receptor $\gamma$ coactivator $1 \beta$ (PGC-1 $\beta$ ), a novel PGC-1-related transcription coactivator associated with host cell factor. J Biol Chem. 2002;277(3):1645-1648.

59. Kressler D, Schreiber SN, Knutti D, Kralli A. The PGC-1-related protein PERC is a selective coactivator of estrogen receptor $\alpha$. J Biol Chem. 2002;277(16):13918-13925

60. Wu Z, et al. Mechanisms controlling mitochondrial biogenesis and respiration through the thermogenic coactivator PGC-1. Cell. 1999;98(1):115-124.

61. Baar K, et al. Adaptations of skeletal muscle to exercise: rapid increase in the transcriptional coactivator PGC-1. FASEB J. 2002;16(14):1879-1886.

62. Terada S, Goto M, Kato M, Kawanaka K, Shimokawa T, Tabata I. Effects of low-intensity prolonged exercise on PGC-1 mRNA expression in rat epitrochlearis muscle. Biochem Biophys Res Commun. 2002;296(2):350-354.

63. Lehman JJ, Barger PM, Kovacs A, Saffitz JE, Medeiros DM, Kelly DP. Peroxisome proliferator-activated receptor $\gamma$ coactivator-1 promotes cardiac mitochondrial biogenesis. J Clin Invest. 2000;106(7):847-856.

64. Scarpulla RC, Vega RB, Kelly DP. Transcriptiona integration of mitochondrial biogenesis. Trends Endocrinol Metab. 2012;23(9):459-466.

65. Ongwijitwat S, Liang HL, Graboyes EM, WongRiley MT. Nuclear respiratory factor 2 senses changing cellular energy demands and its silencing down-regulates cytochrome oxidase and other target gene mRNAs. Gene. 2006;374:39-49.

66. Dhar SS, Ongwijitwat S, Wong-Riley MT. Nuclear respiratory factor 1 regulates all ten nuclearencoded subunits of cytochrome c oxidase in neurons. J Biol Chem. 2008;283(6):3120-3129.

67. Scarpulla RC. Nucleus-encoded regulators of mitochondrial function: integration of respiratory chain expression, nutrient sensing and metabolic stress. Biochim Biophys Acta. 2012; 1819(9-10):1088-1097.

68. Russell LK, et al. Cardiac-specific induction of the transcriptional coactivator peroxisome proliferator-activated receptor $\gamma$ coactivator- $1 \alpha$ promotes mitochondrial biogenesis and reversible cardiomyopathy in a developmental stage-dependent manner. Circ Res. 2004;94(4):525-533.

69. Leone TC, et al. PGC-1 $\alpha$ deficiency causes multi-system energy metabolic derangements: muscle dysfunction, abnormal weight control and hepatic steatosis. PLoS Biol. 2005;3(4):e101.

70. Lai L, et al. Transcriptional coactivators PGC$1 \alpha$ and PGC- $1 \beta$ control overlapping programs required for perinatal maturation of the heart. 
Genes Dev. 2008;22(14):1948-1961

71. Martin OJ, et al. A role for peroxisome proliferator-activated receptor $\gamma$ coactivator- 1 in the control of mitochondrial dynamics during postnatal cardiac growth. Circ Res. 2014;114(4):626-636.

72. Lehman JJ, et al. The transcriptional coactivator PGC-1alpha is essential for maximal and efficient cardiac mitochondrial fatty acid oxidation and lipid homeostasis. Am J Physiol Heart Circ Physiol. 2008;295(1):H185-H196.

73. Arany Z, Novikov M, Chin S, Ma Y, Rosenzweig A, Spiegelman BM. Transverse aortic constriction leads to accelerated heart failure in mice lacking PPAR- $\gamma$ coactivator $1 \alpha$. Proc Natl Acad Sci US A. 2006;103(26):10086-10091.

74. Riehle C, et al. PGC-1 $\beta$ deficiency accelerates the transition to heart failure in pressure overload hypertrophy. Circ Res. 2011;109(7):783-793.

75. Soriano FX, Liesa M, Bach D, Chan DC, Palacín M, Zorzano A. Evidence for a mitochondrial regulatory pathway defined by peroxisome proliferator-activated receptor- $\gamma$ coactivator- $1 \alpha$, estrogen-related receptor- $\alpha$, and mitofusin 2. Diabetes. 2006;55(6):1783-1791.

76. Lai L, et al. A role for peroxisome proliferator-activated receptor $\gamma$ coactivator 1 (PGC-1) in the regulation of cardiac mitochondrial phospholipid biosynthesis. J Biol Chem. 2014;289(4):2250-2259.

77. Shirihai OS, Song M, Dorn GW. How mitochondrial dynamism orchestrates mitophagy. Circ Res. 2015;116(11):1835-1849.

78. Lee S, et al. Mitofusin 2 is necessary for striatal axonal projections of midbrain dopamine neurons. Hum Mol Genet. 2012;21(22):4827-4835.

79. Chen Y, Dorn GW. PINK1-phosphorylated mitofusin 2 is a Parkin receptor for culling damaged mitochondria. Science. 2013;340(6131):471-475.

80. Gong G, Song M, Csordas G, Kelly DP, Matkovich SJ, Dorn GW. Parkin-mediated mitophagy directs perinatal cardiac metabolic maturation in mice. Science. 2015;350(6265): $\operatorname{aad} 2459$.

81. Miyata S, Minobe W, Bristow MR, Leinwand LA. Myosin heavy chain isoform expression in the failing and nonfailing human heart. Circ Res. 2000;86(4):386-390.

82. Allard MF, Schönekess BO, Henning SL, English DR, Lopaschuk GD. Contribution of oxidative metabolism and glycolysis to ATP production in hypertrophied hearts. Am J Physiol. 1994; 267(2 pt 2):H742-H750.

83. Sack MN, Rader TA, Park S, Bastin J, McCune SA, Kelly DP. Fatty acid oxidation enzyme gene expression is downregulated in the failing heart. Circulation. 1996;94(11):2837-2842.

84. Dávila-Román VG, et al. Altered myocardial fatty acid and glucose metabolism in idiopathic dilated cardiomyopathy. J Am Coll Cardiol. 2002;40(2):271-277.

85. Neglia D, et al. Impaired myocardial metabolic reserve and substrate selection flexibility during stress in patients with idiopathic dilated cardiomyopathy. Am J Physiol Heart Circ Physiol. 2007;293(6):H3270-H3278.

86. Kadkhodayan A, Coggan AR, Peterson LR. A "PET" area of interest: myocardial metabolism in human systolic heart failure. Heart Fail Rev. 2013;18(5):567-574.

87. Lai L, et al. Energy metabolic reprogramming in the hypertrophied and early stage failing heart: a multisystems approach. Circ Heart Fail. 2014;7(6):1022-1031.

88. Aubert G, et al. The failing heart relies on ketone bodies as a fuel. Circulation. 2016;133(8):698-705

89. Bedi KC, et al. Evidence for intramyocardial disruption of lipid metabolism and increased myocardial ketone utilization in advanced human heart failure. Circulation. 2016;133(8):706-716.

90. Barger PM, Brandt JM, Leone TC, Weinheimer CJ, Kelly DP. Deactivation of peroxisome proliferator-activated receptor- $\alpha$ during cardiac hypertrophic growth. JClin Invest. 2000;105(12):1723-1730.

91. Kanda H, Nohara R, Hasegawa K, Kishimoto C, Sasayama S. A nuclear complex containing $\mathrm{PPAR} \alpha / \mathrm{RXR} \alpha$ is markedly downregulated in the hypertrophied rat left ventricular myocardium with normal systolic function. Heart Vessels. 2000;15(4):191-196.

92. Barger PM, Browning AC, Garner AN, Kelly DP.p38 mitogen-activated protein kinase activates peroxisome proliferator-activated receptor $\alpha$ : a potential role in the cardiac metabolic stress response. J Biol Chem. 2001;276(48):44495-44501.

93. Huss JM, Levy FH, Kelly DP. Hypoxia inhibits the peroxisome proliferator-activated receptor $\alpha$ / retinoid $\mathrm{X}$ receptor gene regulatory pathway in cardiac myocytes: a mechanism for O2-dependent modulation of mitochondrial fatty acid oxidation. J Biol Chem. 2001;276(29):27605-27612.

94. Pellieux C, et al. Overexpression of angiotensinogen in the myocardium induces downregulation of the fatty acid oxidation pathway. J Mol Cell Cardiol. 2006;41(3):459-466.

95. Pellieux C, Montessuit C, Papageorgiou I, Lerch R. Angiotensin II downregulates the fatty acid oxidation pathway in adult rat cardiomyocytes via release of tumour necrosis factor- $\alpha$. Cardiovasc Res. 2009;82(2):341-350.

96. Lahey R, Wang X, Carley AN, Lewandowski ED. Dietary fat supply to failing hearts determines dynamic lipid signaling for nuclear receptor activation and oxidation of stored triglyceride. Circulation. 2014;130(20):1790-1799.

97. Kolwicz SC Jr, et al. Cardiac-specific deletion of acetyl CoA carboxylase 2 prevents metabolic remodeling during pressure-overload hypertrophy. Circ Res. 2012;111(6):728-738.

98. Szczepaniak LS, et al. Myocardial triglycerides and systolic function in humans: in vivo evaluation by localized proton spectroscopy and cardiac imaging. Magn Reson Med. 2003;49(3):417-423.

99. McGavock JM, et al. Cardiac steatosis in diabetes mellitus: a 1H-magnetic resonance spectroscopy study. Circulation. 2007;116(10):1170-1175.

100.O'Connor RD, Bashir A, Todd Cade W, Yarasheski KE, Gropler RJ. 1H-magnetic resonance spectroscopy for quantifying myocardial lipid content in humans with the cardiometabolic syndrome. J Clin Hypertens (Greenwich). 2009;11(9):528-532.

101. Rijzewijk LJ, et al. Myocardial steatosis is an independent predictor of diastolic dysfunction in type 2 diabetes mellitus. J Am Coll Cardiol. 2008;52(22):1793-1799.

102. Korosoglou G, et al. Left ventricular diastolic function in type 2 diabetes mellitus is associated with myocardial triglyceride content but not with impaired myocardial perfusion reserve. JMagn
Reson Imaging. 2012;35(4):804-811.

103. Levelt $\mathrm{E}$, et al. Relationship between left ventricular structural and metabolic remodeling in type 2 diabetes. Diabetes. 2016;65(1):44-52.

104. Rijzewijk LJ, et al. Altered myocardial substrate metabolism and decreased diastolic function in nonischemic human diabetic cardiomyopathy: studies with cardiac positron emission tomography and magnetic resonance imaging. J Am Coll Cardiol. 2009;54(16):1524-1532.

105. Peterson LR, et al. Sex and type 2 diabetes: obesity-independent effects on left ventricular substrate metabolism and relaxation in humans. Obesity (Silver Spring). 2012;20(4):802-810.

106. Finck BN, et al. A critical role for PPAR $\alpha$-mediated lipotoxicity in the pathogenesis of diabetic cardiomyopathy: modulation by dietary fat content. Proc Natl Acad Sci U S A. 2003;100(3):1226-1231.

107. Georgiadi A, et al. Induction of cardiac Angptl4 by dietary fatty acids is mediated by peroxisome proliferator-activated receptor beta/delta and protects against fatty acid-induced oxidative stress. Circ Res. 2010;106(11):1712-1721.

108. Son NH, et al. Cardiomyocyte expression of PPARgamma leads to cardiac dysfunction in mice. JClin Invest. 2007;117(10):2791-2801.

109. Son NH, et al. PPAR $\gamma$-induced cardiolipotoxicity in mice is ameliorated by PPAR $\alpha$ deficiency despite increases in fatty acid oxidation. J Clin Invest. 2010;120(10):3443-3454.

110. Jun M, et al. Effects of fibrates on cardiovascular outcomes: a systematic review and meta-analysis. Lancet. 2010;375(9729):1875-1884.

111. Soto P, et al. The PPAR $\alpha$ activator fenofibrate fails to alter myocardial metabolism in healthy individuals despite marked peripheral effects. J Nucl Med. 2009;50(supplement 2):419.

112. Neubauer S, et al. Myocardial phosphocreatine-to-ATP ratio is a predictor of mortality in patients with dilated cardiomyopathy. Circulation. 1997;96(7):2190-2196.

113. Ingwall JS, Weiss RG. Is the failing heart energy starved? On using chemical energy to support cardiac function. Circ Res. 2004;95(2):135-145.

114. Jullig M, et al. Is the failing heart out of fuel or a worn engine running rich? A study of mitochondria in old spontaneously hypertensive rats. Proteomics. 2008;8(12):2556-2572.

115. Bugger $\mathrm{H}$, et al. Proteomic remodelling of mitochondrial oxidative pathways in pressure overload-induced heart failure. Cardiovasc Res. 2010;85(2):376-384.

116. Abel ED, Doenst T. Mitochondrial adaptations to physiological vs. pathological cardiac hypertrophy. Cardiovasc Res. 2011;90(2):234-242.

117. Gupte AA, et al. Mechanical unloading promotes myocardial energy recovery in human heart failure. Circ Cardiovasc Genet. 2014;7(3):266-276.

118. Garnier A, Fortin D, Deloménie C, Momken I, Veksler V, Ventura-Clapier R. Depressed mitochondrial transcription factors and oxidative capacity in rat failing cardiac and skeletal muscles. J Physiol (Lond). 2003;551(pt 2):491-501.

119. Sihag S, Cresci S, Li AY, Sucharov CC, Lehman JJ. PGC-1alpha and ERRalpha target gene downregulation is a signature of the failing human heart. JMol Cell Cardiol. 2009;46(2):201-212.

120.Karamanlidis G, Nascimben L, Couper GS, Shekar 
PS, del Monte F, Tian R. Defective DNA replication impairs mitochondrial biogenesis in human failing hearts. Circ Res. 2010;106(9):1541-1548.

121. Chokshi A, et al. Ventricular assist device implantation corrects myocardial lipotoxicity, reverses insulin resistance, and normalizes cardiac metabolism in patients with advanced heart failure. Circulation. 2012;125(23):2844-2853.

122. Bello NA, Arany Z. Molecular mechanisms of peripartum cardiomyopathy: a vascular/ hormonal hypothesis. Trends Cardiovasc Med. 2015;25(6):499-504.
123. Patten IS, et al. Cardiac angiogenic imbalance leads to peripartum cardiomyopathy. Nature. 2012;485(7398):333-338.

124.Sack MN, Disch DL, Rockman HA, Kelly DP. A role for Sp and nuclear receptor transcription factors in a cardiac hypertrophic growth program. Proc Natl Acad Sci U S A. 1997;94(12):6438-6443.

125. Wu SP, et al. Increased COUP-TFII expression in adult hearts induces mitochondrial dysfunction resulting in heart failure. Nat Commun. 2015;6:8245.

126. Rosell M, Jones MC, Parker MG. Role of nuclear receptor corepressor RIP140 in metabolic syndrome. Biochim Biophys Acta. 2011;1812(8):919-928.

127. Chen Y, et al. Roles of transcriptional corepressor RIP140 and coactivator PGC-1 $\alpha$ in energy state of chronically infarcted rat hearts and mitochondrial function of cardiomyocytes. Mol Cell Endocrinol. 2012;362(1-2):11-18.

128. Fritah A, et al. Elevated expression of the metabolic regulator receptor-interacting protein 140 results in cardiac hypertrophy and impaired cardiac function. Cardiovasc Res. 2010;86(3):443-451. 